\title{
Intersection numbers with Witten's top Chern class
}

\author{
SERGEY SHADRIN \\ DIMITRI ZVONKINE
}

\begin{abstract}
Witten's top Chern class is a particular cohomology class on the moduli space of Riemann surfaces endowed with $r$-spin structures. It plays a key role in Witten's conjecture relating to the intersection theory on these moduli spaces.

Our first goal is to compute the integral of Witten's class over the so-called double ramification cycles in genus 1 . We obtain a simple closed formula for these integrals. This allows us, using the methods of the first author [18], to find an algorithm for computing the intersection numbers of the Witten class with powers of the $\psi$-classes over any moduli space of $r$-spin structures, in short, all numbers involved in Witten's conjecture.
\end{abstract}

$14 \mathrm{H} 10 ; 14 \mathrm{H} 70$

\section{Introduction}

\subsection{Aims and purposes}

In $1991 \mathrm{E}$ Witten formulated two conjectures relating to the intersection theory of moduli spaces of curves $[19 ; 20]$, motivated by two dimensional gravity.

The first conjecture involves moduli spaces of stable curves and certain 2-cohomology classes on them, called $\psi$-classes. The intersection numbers of powers of the $\psi-$ classes can be arranged into a generating series that is claimed to be a solution of the Korteweg-de Vries (or KdV) hierarchy of partial differential equations. This conjecture was first proved by M Kontsevich in [12]. At present there are several alternative proofs by Okounkov and Pandharipande [15], Mirzakhani [13], Kim and Liu [11], Kazarian and Lando [10], Chen, Li and Liu [2] and Kazarian [9].

The second conjecture ${ }^{1}$ involves a more complicated moduli space, called the space of $r$-spin structures. Apart from the $\psi$-classes, one considers one more cohomology class, called the Witten top Chern class, or just Witten's class for shortness. We are

\footnotetext{
${ }^{1}$ It was recently proved by C Faber and the authors [6], two years after the completion of the present work.
} 
interested in the intersection numbers of Witten's class with powers of the $\psi$-classes. These intersection numbers can, once again, be arranged into a generating series, and this series is a solution of the $r$-th Gelfand-Dikii (or the $r-\mathrm{KdV}$ ) hierarchy.

Giving a proper formulation of this conjecture required joint work by several people (references are given below). In particular, the precise definition of Witten's top Chern class is rather involved. However this class is known to satisfy quite simple factorization rules.

In [18] the first author found an "almost algorithm" for computing some of the intersection numbers arising in Witten's second conjecture using only the factorization rules for the Witten class. More precisely, the factorization rules allow one to express more complicated intersection numbers via simpler ones, until one arrives at unsimplifiable cases. These can be of two types: (i) Integrals of Witten's class (with no $\psi$-classes) over genus 0 moduli spaces. These numbers are well-known. (ii) Integrals of Witten's class (with no $\psi$-classes) over some special divisors on genus 1 moduli space. (These divisors have a rather cumbersome name of double ramification divisors - see Definitions 1.1 and 2.4.) When numbers of second type appeared in the course of computations the algorithm blocked without giving an answer.

The purpose of this note is twofold.

First, we compute the integrals of Witten's class over the double ramification divisors in genus 1. It turns out that a simple closed formula exists for these integrals.

Second, we complete and give a coherent exposition of the algorithm for computing Witten's intersection numbers. Our computation uses only factorization rules for Witten's class. Therefore, we obtain the following theorem ${ }^{2}$.

Theorem 1 The intersection numbers of Witten's class with powers of the $\psi$-classes are entirely determined by (i) genus 0 intersection numbers involving no $\psi$-classes, and (ii) the factorization rules for Witten's class.

\subsection{Main definitions}

1.2.1 Moduli spaces $\mathcal{M}_{g, n}$ is the moduli space of smooth complex genus $g$ curves with $n \geq 1$ distinct numbered marked points. $\overline{\mathcal{M}}_{g, n}$ is its Deligne-Mumford compactification, in other words, the moduli space of stable curves. Over $\overline{\mathcal{M}}_{g, n}$ we define $n$ holomorphic line bundles $\mathcal{L}_{i}$. The fiber of $\mathcal{L}_{i}$ over a point $q \in \overline{\mathcal{M}}_{g, n}$ is the cotangent line to the corresponding stable curve $C_{q}$ at the $i$-th marked point. We denote by $\psi_{i}=c_{1}\left(\mathcal{L}_{i}\right)$ their first Chern classes.

\footnotetext{
${ }^{2}$ In [6] another proof of Theorem 1 is given; it is simpler, but less constructive.
} 
1.2.2 Spaces of $r$-spin structures Choose an integer $r \geq 2$ and pick $n$ integers $a_{1}, \ldots a_{n} \in\{0, \ldots, r-1\}$ in such a way that $2 g-2-\sum a_{i}$ is divisible by $r$. The numbers $a_{1}, \ldots, a_{n}$ are assigned to the marked points $x_{1}, \ldots, x_{n}$. On a smooth curve $C$ one can find $r^{2 g}$ different line bundles $\mathcal{T}$ with an identification

$$
\mathcal{T}^{\otimes r} \stackrel{\sim}{\rightarrow} K\left(-\sum a_{i} x_{i}\right) .
$$

The space of smooth curves endowed with such a line bundle $\mathcal{T}$ is called the space of $r$-spin structures and denoted by $\mathcal{M}_{g ; a_{1}, \ldots, a_{n}}^{1 / r}$. Every $r$-spin structure $\mathcal{T}$ has $r$ "trivial" automorphisms given by the multiplication by $r$-th roots of unity along the fibers of $\mathcal{T}$. Therefore the stabilizer of the generic point of the moduli stack is $\mathbb{Z} / r \mathbb{Z}$. However, we choose to work with the rigidified moduli space factoring out $\mathbb{Z} / r \mathbb{Z}$ in all stabilizers. Thus $\mathcal{M}_{g ; a_{1}, \ldots, a_{n}}^{1 / r}$ is an unramified $r^{2 g}$-sheeted covering of $\mathcal{M}_{g, n}$. A compactification of this space, denoted by $\overline{\mathcal{M}}_{g ; a_{1}, \ldots, a_{n}}^{1 / r}$, was constructed by Jarvis alone [8] and with Abramovich [1] (see also Chiodo [3] for a simplified version). It is a smooth orbifold (or stack), and there is a finite projection mapping of degree $r^{2 g}$

$$
p: \overline{\mathcal{M}}_{g ; a_{1}, \ldots, a_{n}}^{1 / r} \rightarrow \overline{\mathcal{M}}_{g, n} .
$$

The simplest construction [3] uses the so-called Abramovich-Vistoli twisted curves, ie, curves that are themselves endowed with an orbifold structure. The stabilizers of the marked points and the nodes are equal to $\mathbb{Z} / r \mathbb{Z}$, while the stabilizer of any other point is trivial. $\mathcal{T}$ is then an $r$-th root of $K\left(-\sum a_{i} x_{i}\right)$ in the orbifold sense. Alternatively, we can forget about the orbifold structure of the curve and consider only the sheaf of invariant sections of $\mathcal{T}$. We then obtain a rank one torsion-free sheaf rather than a line bundle, which leads to the construction of the compactification given in [8].

1.2.3 Witten's class The rank one torsion-free sheaf (of invariant sections of) $\mathcal{T}$ is defined on the universal curve $\overline{\mathcal{C}}_{g ; a_{1}, \ldots, a_{n}}^{1 / r}$ over $\overline{\mathcal{M}}_{g ; a_{1}, \ldots, a_{n}}^{1 / r}$. Consider its pushforward to the space $\overline{\mathcal{M}}_{g ; a_{1}, \ldots, a_{n}}^{1 / r}$ itself. First assume that for each curve $C$ we have $H^{0}(C, \mathcal{T})=0$. Then the spaces $H^{1}(C, \mathcal{T})$ form a vector bundle $V^{\vee}$ over $\overline{\mathcal{M}}_{g ; a_{1}, \ldots, a_{n}}^{1 / r}$. We denote by $V$ the dual vector bundle and define Witten's class as

$$
c_{W}\left(a_{1}, \ldots, a_{n}\right)=c_{W}=\frac{1}{r^{g}} p_{*} c_{\mathrm{top}}(V) .
$$

In other words: take the top Chern class of $V$, push it from $\overline{\mathcal{M}}_{g ; a_{1}, \ldots, a_{n}}^{1 / r}$ to $\overline{\mathcal{M}}_{g, n}$, and divide by $r^{g}$. By the Riemann-Roch formula, the (complex) degree of Witten's class is

$$
\operatorname{deg} c_{W}=\frac{(r-2)(g-1)+\sum a_{i}}{r} .
$$

Unfortunately, in general $\mathcal{T}$ has both 0 - and 1-cohomologies. The definition of Witten's class $c_{W}$ in this case is much more involved. There exist two compatible 
algebro-geometric constructions: see Polishchuk and Vaintrob [17] and Chiodo [4]. (Note that the definition of $c_{W}$ is special to our situation and uses the identification of $\mathcal{T}^{\otimes r}$ with the canonical bundle. No general constructions from algebraic geometry are expected to work.)

Witten's class satisfies the following vanishing property, that we will use as an axiom:

$$
\text { If one of the } a_{i} \text { 's equals } r-1 \text { then } c_{W}=0 .
$$

1.2.4 Factorization rules We are interested in the restriction of Witten's class to the boundary components of the moduli space $\overline{\mathcal{M}}_{g, n}$. There are two types of boundary components (see Figure 1): those isomorphic to $\overline{\mathcal{M}}_{g^{\prime}, n^{\prime}+1} \times \overline{\mathcal{M}}_{g^{\prime \prime}, n^{\prime \prime}+1}, n^{\prime}+n^{\prime \prime}=n$, $g^{\prime}+g^{\prime \prime}=g$ and the unique component isomorphic to $\overline{\mathcal{M}}_{g-1, n+2} / \mathbb{Z}_{2}$.
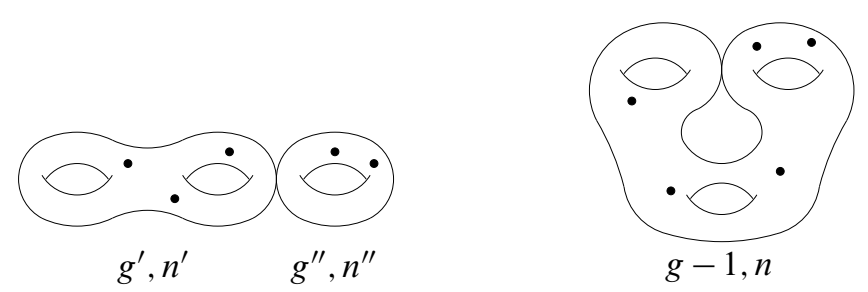

Figure 1: Two possible degenerations of a stable curve

In the first case, assume for simplicity that the marked points $x_{1}, \ldots, x_{n^{\prime}}$ are on the first component of the curve, while $x_{n^{\prime}+1}, \ldots, x_{n}$ are on the second component. There is a unique choice of $a^{\prime}, a^{\prime \prime} \in\{0, \ldots, r-1\}$ such that

$$
2 g^{\prime}-2-a^{\prime}-\sum_{i=1}^{n^{\prime}} a_{i} \quad \text { and } \quad 2 g^{\prime \prime}-2-a^{\prime \prime}-\sum_{i=n^{\prime}+1}^{n} a_{i}
$$

are both divisible by $r$. We have $a^{\prime}+a^{\prime \prime}=r-2$ or $a^{\prime}=a^{\prime \prime}=r-1$. For the second type of boundary component, we have to sum over all choices of $a^{\prime}, a^{\prime \prime}$ such that $a^{\prime}+a^{\prime \prime}=r-2$. Now we can formulate the factorization rules.

The restriction of Witten's class to the first type boundary component equals

$$
c_{W}\left(a_{1}, \ldots, a_{n}\right)=c_{W}\left(a_{1}, \ldots, a_{n^{\prime}}, a^{\prime}\right) \times c_{W}\left(a_{n^{\prime}+1}, \ldots, a_{n}, a^{\prime \prime}\right) .
$$

The restriction of Witten's class to the second type boundary component equals

$$
c_{W}\left(a_{1}, \ldots, a_{n}\right)=\frac{1}{2} \sum_{a^{\prime}+a^{\prime \prime}=r-2} c_{W}\left(a_{1}, \ldots, a_{n}, a^{\prime}, a^{\prime \prime}\right) .
$$


The vanishing property and the factorization rules (for the Polishchuk-Vaintrob construction) are proved in [16].

1.2.5 Intersection numbers We use the standard notation for the intersection numbers of Witten's class with powers of the $\psi$-classes:

$$
\left\langle\tau_{d_{1}, a_{1}} \ldots \tau_{d_{n}, a_{n}}\right\rangle=\int_{\overline{\mathcal{M}}_{g, n}} c_{W}\left(a_{1}, \ldots, a_{n}\right) \psi_{1}^{d_{1}} \ldots \psi_{n}^{d_{n}} .
$$

Although the genus $g$ is determined by the elements of the bracket by

$$
(r+1)(2 g-2+n)=\sum\left(r d_{i}+a_{i}+1\right),
$$

we will sometimes recall it in a subscript. The integer $r \geq 2$ is supposed to be fixed once and for all throughout the paper.

Since double ramification cycles on genus 1 moduli spaces will play a special role, let us introduce them here (this is a particular case of Definition 2.4). Choose $n$ integers $k_{1}, \ldots, k_{n}$ satisfying $\sum k_{i}=0$. We assume that at least one of the $k_{i}$ 's is different from 0 .

We will usually assume that the list $\left(k_{1}, \ldots, k_{n}\right)$ starts with the positive integers and ends with the negative ones, the zeroes being in the middle. We will sometimes use the notation

$$
\left(k_{1}, \ldots, k_{n_{+}}|0, \ldots, 0| \tilde{k}_{1}, \ldots, \tilde{k}_{n_{-}}\right)
$$

with only nonnegative integers instead of

$$
\left(k_{1}, \ldots, k_{n}\right)=\left(k_{1}, \ldots, k_{n_{+}}, 0, \ldots, 0,-\tilde{k}_{1}, \ldots,-\tilde{k}_{n_{-}}\right) .
$$

To this list of integers assign the set $D\left(k_{1}, \ldots, k_{n}\right)$ of smooth genus 1 curves $\left(C, x_{1}, \ldots, x_{n}\right)$ such that $\sum k_{i} x_{i}$ is the divisor of a function on $C$. Let the cycle $\bar{D}\left(k_{1}, \ldots, k_{n}\right)$ be the closure of $D\left(k_{1}, \ldots, k_{n}\right)$ in $\overline{\mathcal{M}}_{1, n}$.

Definition 1.1 We call $\bar{D}\left(k_{1}, \ldots, k_{n}\right)$ a double ramification cycle. The integral of Witten's class over this cycle is denoted by

$$
\begin{aligned}
& \int c_{W}\left(a_{1}, \ldots, a_{n}\right)=\left\langle\begin{array}{ccc}
k_{1} & \ldots & k_{n} \\
a_{1} & \ldots & a_{n}
\end{array}\right\rangle \\
& \bar{D}\left(k_{1}, \ldots, k_{n}\right) \\
& =\left\langle\begin{array}{ccc|ccc|ccc}
k_{1} & \ldots & k_{n_{+}} & 0 & \ldots & 0 & \tilde{k}_{1} & \ldots & \tilde{k}_{n_{-}} \\
a_{1} & \ldots & \ldots & \ldots & \ldots & \ldots & \ldots & \ldots & a_{n}
\end{array}\right\rangle .
\end{aligned}
$$


Theorem 2 The integral of Witten's class $c_{W}\left(a_{1}, \ldots, a_{n}\right)$ over the double ramification cycle $\bar{D}\left(k_{1}, \ldots, k_{n}\right) \subset \overline{\mathcal{M}}_{1, n}$ equals

$$
\left\langle\begin{array}{lll}
k_{1} & \ldots & k_{n} \\
a_{1} & \ldots & a_{n}
\end{array}\right\rangle_{g=1}=\left(\frac{1}{2} \sum_{i=1}^{n} k_{i}^{2}-1\right) \cdot \frac{1}{24} \frac{(n-1) !}{r^{n-1}} \prod_{i=1}^{n}\left(r-1-a_{i}\right)
$$

if $\sum a_{i}=r(n-1)$ and vanishes otherwise.

Acknowledgements This work was completed during the second author's visit to the Stockholm University. We would like to thank the University for its hospitality and the members of the mathematical seminar for their interest. We thank A Vainshtein for pointing out a mistake in our initial proof of Proposition 4.1. We are grateful to the unknown referee for a very careful reading and many useful remarks.

The first author was partly supported by the grants NSh-1972.2003.1, NWO-RFBR047.011.2004.026. The second author was partly supported by the ANR project "Geometry and Integrability in Mathematical Physics".

\section{Preliminaries}

\subsection{Admissible coverings}

Consider a map $\varphi$ from a smooth curve $C$ to the sphere $S=\mathbb{C P}^{1}$. On $S$, introduce a (finite) set of marked points containing all the branch points of $\varphi$. On $C$, mark all the preimages of the marked points on $S$. Now choose several disjoint simple loops on $S$, that do not pass through the marked points. Suppose that if we contract these loops we obtain a stable genus 0 curve $S^{\prime}$. Now contract also all the preimages of the loops in $C$ to obtain a nodal curve $C^{\prime}$ that turns out to be automatically stable. We have obtained a map $\varphi^{\prime}$ from a nodal curve of genus $g$ to a stable curve of genus 0 . It has the same degree over every component of $S^{\prime}$. Moreover, at each node of $C^{\prime}$, the projection $\varphi^{\prime}$ has the same local multiplicity on both components meeting at the node.

Definition 2.1 A map from a stable curve of genus $g$ to a stable curve of genus 0 topologically equivalent to a map described above is called an admissible covering.

The space of all admissible coverings with prescribed ramification types over the marked points is very useful for the study of moduli spaces [7]. It is not normal but can be normalized, and thus one can study its intersection theory. We refer to Ionel's work [7] for detailed definitions. 
We will be particularly interested in the space of admissible coverings with multiple ramifications only over 2 points labeled with 0 and $\infty$, the other ramification points being simple.

An admissible covering induces a map of finite sets

$$
\widehat{\varphi}:(\text { set of labels on } C) \rightarrow(\text { set of labels on } S) \text {. }
$$

Although we will not stress this point every time, we will always assume that both sets of labels of the marked points and the map $\hat{\varphi}$ are given once and for all. In other words, if we choose a marked points on $C$, we know automatically the label of its image on $S$ and, conversely, if we choose a marked point on $S$ we know the set of labels of its preimages.

Definition 2.2 Consider the space of admissible coverings of some given genus $g$ with prescribed ramification types $\left(k_{1}, \ldots, k_{n_{+}}\right)$and $\left(\tilde{k}_{1}, \ldots, \widetilde{k}_{n_{-}}\right), \sum k_{i}=\sum \widetilde{k_{i}}$, over two points labeled 0 and $\infty$, simple ramifications over $n_{+}+n_{-}+2 g-2$ more points of $S$, and, finally, $n_{0}$ additional marked points on $S$. The normalization of this space is called a double ramification space or a DR-space. It is denoted by

$$
\overline{\mathbf{A}}=\overline{\mathbf{A}}(k_{1}, \ldots, k_{n_{+}}, \overbrace{0, \ldots, 0}^{n_{0}},-\tilde{k}_{1}, \ldots,-\tilde{k}_{n_{-}}) .
$$

We do not include the genus $g$ in our notation, although, of course, the space of coverings does depend on $g$.

Let $K=\sum k_{i}=\sum \tilde{k}_{i}$ and $n=n_{+}+n_{-}+n_{0}$. Then the total number of marked points on the curve $C$ equals $N=n K+(2 g-2)(K-1)$. We can consider the forgetful map $j: \overline{\mathbf{A}} \rightarrow \overline{\mathcal{M}}_{g, N}$ that forgets the admissible covering retaining only its source curve. Since the curve $C$ is automatically stable, the map $j$ is actually an injection and an isomorphism with its image ${ }^{3}$. The pullbacks by $j$ of the $\psi$-classes on $\overline{\mathcal{M}}_{g, N}$ coincide with the $\psi$-classes naturally defined on $\overline{\mathbf{A}}$.

Another forgetful map $f: \overline{\mathbf{A}} \rightarrow \overline{\mathcal{M}}_{0, n+2 g}$ takes an admissible covering to its image genus 0 curve. This map satisfies the following crucial property.

\footnotetext{
${ }^{3}$ Indeed, suppose the curve $C$ is given. Here is how we reconstruct the admissible covering $\varphi$. First, the topological structure of the image curve $S$ of $\varphi$ is determined by the following condition: two marked points lie on different components whenever they have two preimages in $C$ lying on different components. (Note that every node of $C$ necessarily separates two different irreducible components.) Once the topological structure of $S$ is known, it is easy to determine which component of $C$ is mapped to which component of $S$ and which node is mapped to which node. Finally, to construct the map $\varphi$ on each component, we note that there are at least three special points on each component of $S$ and that a meromorphic function is uniquely determined by its zeroes, poles, and one more point at which its value is known.
}

Geometry 8 Topology, Volume 12 (2008) 
Lemma 2.3 (Ionel [7, Lemma 1.17]) The map $f$ is finite. We have

$$
\psi_{i}(\overline{\mathbf{A}})=\frac{1}{k_{i}} \psi_{0}\left(\overline{\mathcal{M}}_{0, n+2 g}\right), \quad \psi_{n_{+}+n_{0}+i}(\overline{\mathbf{A}})=\frac{1}{\widetilde{k}_{i}} \psi_{\infty}\left(\overline{\mathcal{M}}_{0, n+2 g}\right) .
$$

In other words, a $\psi$-class on $\overline{\mathbf{A}}$ at a marked zero or pole of the admissible covering coincides, up to a constant, with the pullback of the $\psi$-class at 0 or $\infty$ of the genus 0 moduli space.

The equality between the $\psi$-classes will be the base of our computations. For the time being, we note two simple consequences of the finiteness of $f$ : first, the dimension of the DR-space equals $n+2 g-3$; second, the set $f^{-1}\left(\mathcal{M}_{0, n+2 g}\right)$ of admissible coverings with smooth source curves $C$ is open dense in the DR-space.

Finally, we define double ramification cycles (this is a generalization of Definition 1.1). For a given moduli space $\overline{\mathcal{M}}_{g, n}$ choose an integer $p, 0 \leq p \leq g$ and $n+p$ integers $k_{i}$. We suppose that $\sum k_{i}=0$, that none of the $k_{n+1}, \ldots, k_{n+p}$ vanishes, and that among $k_{1}, \ldots, k_{n}$ there is at least one positive and at least one negative integer.

Definition 2.4 Let $D_{g, n}\left(k_{1}, \ldots, k_{n+p}\right)$ be the set of smooth curves $\left(C, x_{1}, \ldots, x_{n}\right)$ in $\mathcal{M}_{g, n}$ such that there exist $p$ more marked points $x_{n+1}, \ldots, x_{n+p}$ and a meromorphic function on $C$ with no zeroes or poles outside of $x_{1}, \ldots, x_{n+p}$, the orders of zeroes or poles being prescribed by the list $k_{1}, \ldots, k_{n+p}\left(k_{i}>0\right.$ for the zeroes, $k_{i}<0$ for the poles, and $k_{i}=0$ for the marked points that are neither zeroes nor poles). The closure of this set in $\overline{\mathcal{M}}_{g, n}$ is called the double ramification cycle or a DR-cycle and denoted by $\bar{D}_{g, n}\left(k_{1}, \ldots, k_{n+p}\right)$.

Recall that the list $\left(k_{1}, \ldots, k_{n+p}\right)$ re-ordered in the nonincreasing order is denoted by

$$
(k_{1}, \ldots, k_{n_{+}}, \overbrace{0, \ldots, 0}^{n_{0}},-\tilde{k}_{1}, \ldots,-\tilde{k}_{n_{-}})
$$

with $n_{+}+n_{0}+n_{-}=n+p$ and $K=\sum_{i=1}^{n_{+}} k_{i}=\sum_{i=1}^{n_{-}} \tilde{k}_{i}$.

Proposition 2.5 If $K=1, g \geq 1$, then the DR-cycle $\bar{D}_{g, n}\left(k_{1}, \ldots, k_{n+p}\right)$ is empty. Else its codimension is equal to $g-p$.

This fact is well-known to the experts, but the complete proof does not appear anywhere in the literature (it is usually referred to as "a generalization of Mumford's argument" [14]). For completeness we present the argument here. 
Proof of Proposition 2.5 $\bar{D}_{g, n}\left(k_{1}, \ldots, k_{n+p}\right)$ is defined as the closure of the set $D_{g, n}\left(k_{1}, \ldots, k_{n+p}\right) \subset \mathcal{M}_{g, n}$; hence it suffices to show that $D_{g, n}\left(k_{1}, \ldots, k_{n+p}\right)$ is of codimension $g-p$ or, in other words, of dimension $2 g-3+n+p$. We deduce the proposition from the three following lemmas.

Lemma 2.6 If $K=1, g \geq 1$, then $D_{g, n}\left(k_{1}, \ldots, k_{n+p}\right)$ is empty. Otherwise $D_{g, n}\left(k_{1}, \ldots, k_{n+p}\right)$ is nonempty and its dimension is at most $2 g-3+n+p$.

The idea of the proof is that $D_{g, n}\left(k_{1}, \ldots, k_{n+p}\right)$ is the image of a so-called projectivized Hurwitz space which is itself of dimension $2 g-3+n+p$.

Lemma 2.7 Let $p=g$. $D_{g, n}\left(k_{1}, \ldots, k_{n}\right)$ is a Zariski open set in $\mathcal{M}_{g, n}$. It is empty if $K=1, g \geq 1$, and dense otherwise.

The idea of the proof is that by moving $g$ points on a genus $g$ curve one can obtain every element of the Jacobian of the curve.

Lemma 2.8 Let $p=0$. Then the codimension of $D_{g, n}\left(k_{1}, \ldots, k_{n}\right)$ is at most $g$.

The idea is that locally $D_{g, n}\left(k_{1}, \ldots, k_{n+g}\right)$ can be given by $g$ equations.

Lemma 2.7 establishes the dimension of $D_{g, n}\left(k_{1}, \ldots, k_{n+p}\right)$ for $p=g$, while Lemmas 2.6 and 2.8 establish the dimension of $D_{g, n}\left(k_{1}, \ldots, k_{n+p}\right)$ for $p=0$. In both cases the dimension equals $2 g-3+n+p$. Consider two forgetful maps $\mathcal{M}_{g, n+p} \rightarrow \mathcal{M}_{g, n} \rightarrow$ $\mathcal{M}_{g, n+p-g}$. These maps send $D_{g, n+p}\left(k_{1}, \ldots, k_{n+p}\right)$ to $D_{g, n}\left(k_{1}, \ldots, k_{n+p}\right)$ and then to $D_{g, n+p-g}\left(k_{1}, \ldots, k_{n+p}\right)$. Since the dimensions of the first and the last spaces are both equal to $2 g-3+n+p$, the dimension of $D_{g, n}\left(k_{1}, \ldots, k_{n+p}\right)$ also equals $2 g-3+n+p$.

Proof of Lemma 2.6 The Hurwitz space $\mathcal{H}_{g ; k_{1}, \ldots, k_{n+p}}$ is the space of ramified coverings of the sphere by (smooth connected) genus $g$ surfaces, with ramification types $\left(k_{1}, \ldots, k_{n_{+}}\right)$and $\left(\tilde{k}_{1}, \ldots, \tilde{k}_{n_{-}}\right)$over 0 and $\infty$ respectively and with $n_{0}$ more marked points on the source curve.

Let $\left(C, f: C \rightarrow \mathbb{C P}^{1}\right)$ be a point of $\mathcal{H}_{g ; k_{1}, \ldots, k_{n+p}}$. On $\mathbb{C} \mathrm{P}^{1}$ mark the branch points of $f$ and the images of the $n_{0}$ marked points on $C$. A generic $f$ has $m=2 g-2+n_{+}+$ $n_{-}$simple branch points, thus in whole there are $m+n_{0}=2 g-2+n+p$ marked points on $\mathbb{C P}^{1}$. (In the nongeneric case, the number of marked points is smaller.) Now, one can reconstruct the couple $(C, f)$ from the set of marked points and some additional discrete information about the monodromies of the ramified covering $f$. Therefore the dimension of $\mathcal{H}_{g ; k_{1}, \ldots, k_{n+p}}$ equals $2 g-2+n+p$. 
Consider the natural forgetful map $\mathcal{H}_{g ; k_{1}, \ldots, k_{n+p}} \rightarrow \mathcal{M}_{g, n}$. The image of this map is $D_{g, n}\left(k_{1}, \ldots, k_{n+p}\right)$. On the other hand, under this map, the dimension drops at least by 1 , since the points $(C, f)$ and $(C, \lambda f)$ have the same image for any $\lambda \in \mathbb{C}^{*}$. Thus the dimension of $D_{g, n}\left(k_{1}, \ldots, k_{n+p}\right)$ is at most $2 g-3+n+p$.

Note that for $g \geq 1$, the case $K=1$ (in other words, $n_{+}=n_{-}=1, k_{1}=\tilde{k}_{1}=1$ ) is an exception: indeed in this case both $\mathcal{H}_{g ; k_{1}, \ldots, k_{n+p}}$ and $D_{g, n}\left(k_{1}, \ldots, k_{n+p}\right)$ are empty, because there are no meromorphic functions of degree 1 on a genus $g \geq 1$ surface. In all other cases, the space $\mathcal{H}_{g ; k_{1}, \ldots, k_{n+p}}$ is nonempty and of dimension $2 g-2+n+p$ as claimed. Indeed, for $K \geq 2$, one can always find in the symmetric group $S_{K}$ two permutations $\sigma_{+}$and $\sigma_{-}$with cycle types $\left(k_{1}, \ldots, k_{n_{+}}\right)$and $\left(\tilde{k}_{1}, \ldots, \widetilde{k}_{n_{-}}\right)$ respectively and $m=2 g-2+n_{+}+n_{-}$transpositions $\tau_{1}, \ldots, \tau_{m}$ such that (i) the product $\sigma_{+} \tau_{m} \ldots \tau_{1} \sigma_{-}$equals the identical permutation; and (ii) the group generated by $\sigma_{+}, \sigma_{-}, \tau_{1}, \ldots, \tau_{m}$ acts transitively on the $K$ elements.

Proof of Lemma 2.7 For simplicity, let us choose a base point in $C$; then every divisor in $C$ determines a point in the jacobian $\operatorname{Jac}(C)$. It is easy to check that the map

$$
\begin{array}{ccc}
C^{g} & \rightarrow & \operatorname{Jac}(C) \\
\left(x_{n+1}, \ldots, x_{n+g}\right) & \mapsto & k_{n+1} x_{1}+\cdots+k_{n+g} x_{g}
\end{array}
$$

is of degree $k_{n+1}^{2} \cdots k_{n+g}^{2} \neq 0$. Therefore, for any curve $C$ and any marked points $x_{1}, \ldots, x_{n}$ there exists a choice of $x_{n+1}, \ldots, x_{n+g}$ such that $\sum_{i=1}^{n+g} k_{i} x_{i}$ is the divisor of a function. Hence the points of $\mathcal{M}_{g, n}$ that do not lie in $D_{g, n}\left(k_{1}, \ldots k_{n+g}\right)$ form a Zariski closed set given by the following Zariski closed condition: if $\sum_{i=1}^{n+g} k_{i} x_{i}$ is the divisor of a function then the points $x_{1}, \ldots x_{n+g}$ are not pairwise distinct.

We have proved that $D_{g, n}\left(k_{1}, \ldots k_{n+g}\right)$ is a Zariski open set. Being the image of the Hurwitz space, it is empty if and only if the Hurwitz space is empty and dense otherwise.

Proof of Lemma 2.8 To simplify the notation, we relabel the $n$ marked points on the curve $C$ by $x_{1}, \ldots x_{n_{+}}, \tilde{x}_{1}, \ldots, \tilde{x}_{n_{-}}, y_{1}, \ldots, y_{n_{0}}$. Over $\mathcal{M}_{g, n}$ we consider two vector bundles $E$ and $F$ of ranks $K+n_{-}+g-1$ and $K+n_{-}+2 g-2$ respectively. The fiber of $E$ over a curve $C \in \mathcal{M}_{g, n}$ is the space of meromorphic differentials with poles of orders $\tilde{k}_{1}+1, \ldots, \tilde{k}_{n_{-}}+1$ at $\tilde{x}_{1}, \ldots, \tilde{x}_{n_{-}}$. The vector bundle $F$ is the sum of $n_{+}+1$ subbundles: $F=H \oplus J_{1} \oplus \cdots \oplus J_{n_{+}}$. Here $J_{i}$ is the bundle of $\left(k_{i}-1\right)$-jets of differentials at $x_{i}$, while

$$
H=H^{1}\left(C \backslash\left\{\tilde{x}_{1}, \ldots, \tilde{x}_{n_{-}}\right\},\left\{x_{1}, \ldots, x_{n_{+}}\right\}, \mathbb{C}\right)
$$


is the first cohomology group of the curve $C$ punctured at the points $\tilde{x}_{1}, \ldots, \tilde{x}_{n_{-}}$and with points $x_{1}, \ldots, x_{n_{+}}$identified. The rank of $J_{i}$ equals $k_{i}-1$, while the rank of $H$ equals $2 g-2+n_{-}+n_{+}$.

There is a natural linear map $v: E \rightarrow F$, assigning to a meromorphic differential from $E$ its jets at the points $x_{i}$ and the cohomology class in $H$ that it represents. Now we claim that the curve $C$ with $n$ marked points belongs to $D_{g, n}\left(k_{1}, \ldots, k_{n}\right)$ if and only if the map $v$ is not injective (ie, has a nontrivial kernel). Indeed, if $\alpha \in \operatorname{ker} v$, then $\alpha$ is an exact differential on $C$ and $f=\int_{x_{1}} \alpha$ has poles of orders $\tilde{k}_{i}$ at $\tilde{x}_{i}$ and zeroes of orders $k_{i}$ at $x_{i}$. Conversely, if $f$ is a function with zeroes and poles like that, then $d f \in \operatorname{ker} v$.

In local coordinates the map $v$ is given by a matrix $\left(K+n_{-}+g-1\right) \times\left(K+n_{-}+\right.$ $2 g-2)$. The condition that this matrix is not of the highest rank is expressed by $g$ equations: the vanishing of $g$ minors. Thus locally, at the neighborhood of any point, $D_{g, n}\left(k_{1}, \ldots, k_{n}\right)$ is given by $g$ equations and has codimension at most $g$.

Faber and Pandharipande [5] proved that the cohomology classes Poincaré dual to any DR-cycle belongs to the tautological ring of the moduli space of curves. Their proof may be used to obtain new relations for intersection numbers with Witten's class.

Introduce the following forgetful map $h: \overline{\mathbf{A}}\left(k_{1}, \ldots, k_{n+p}\right) \rightarrow \overline{\mathcal{M}}_{g, n}$. Take an admissible covering $\varphi: C \rightarrow S$. Forget the covering itself, retaining only the source curve $C$. Further, on $C$, forget all the preimages of the branch points on $S$, except 0 and $\infty$. Forget those preimages of 0 and $\infty$ that correspond to $k_{n+1}, \ldots, k_{n+p}$, but retain the $n_{+}+n_{-}$others. For each of the $n_{0}$ additional marked points of $S$, retain exactly one of its preimages (say, the one with the smallest number) and forget the others. Finally, stabilize the curve thus obtained. We have a obtained a stable curve $C$ with $n$ marked points naturally labeled by $k_{1}, \ldots, k_{n}$. This curve is the image of the initial admissible covering under the map $h$.

Proposition 2.9 The map $h$ sends the fundamental homology class of $\overline{\mathbf{A}}\left(k_{1}, \ldots, k_{n+p}\right)$ to a multiple of the fundamental homology class of the DR-cycle $\bar{D}_{g, n}\left(k_{1}, \ldots, k_{n+p}\right)$.

Proof This follows from Ionel's lemma and Proposition 2.5. Indeed, both spaces have the same dimension $(n+p+2 g)-3=3 g-3+n-(g-p)$. Moreover, $h$ is surjective because, by definition, every point in the open part of $\bar{D}_{g, n}\left(k_{1}, \ldots, k_{n+p}\right)$ gives rise to a unique admissible covering up to the numbering of the marked points. 
Conventions By now we have introduced several spaces and forgetful maps between them. We sum up these definitions in Figure 2.

The figure represents a DR-space

$$
\overline{\mathbf{A}}\left(k_{1}+1, \ldots, k_{n_{+}}, 0, \ldots, 0,-\tilde{k}_{1}, \ldots,-\tilde{k}_{n_{-}},-1\right) .
$$

for $g=1$. If we denote by $K$ the sum $\sum k_{i}=\sum \tilde{k}_{i}$, the total number of marked points on $C$ equals $N=(n+1)(K+1)$. The figure also shows the forgetful maps $j, f$ and $h$. In this figure, as well as in the subsequent figures and in the text we follow the following conventions. A cross represents a critical point or a branch point of an admissible covering. Round black dots represent the marked points $x_{1}, \ldots, x_{n}$ (they are not forgotten under the map $h$ ). The images of these points under the maps $j, f$, and $h$ are also represented as round black dots. Square black dots represent the points $x_{n+1}, \ldots, x_{n+p}$ (they are forgotten under the map $h$ ). Finally, white dots represent all the marked points on the curve $C$ different from the critical points and from $x_{1}, \ldots, x_{n+p}$. We will not show them in the figures when it is not necessary.

\subsection{Intersection numbers in genus 0}

In our computations we will need the value of the bracket

$$
\left\langle\tau_{1, a_{1}} \tau_{0, a_{2}} \ldots \tau_{0, a_{n}}\right\rangle_{1}=\int_{\overline{\mathcal{M}}_{g ; a_{1}, \ldots, a_{n}}^{1 / r}} c_{W} \psi_{1}
$$

for $\sum a_{i}=(n-1) r$. The topological recursion relation expresses $\psi_{1}$ as a sum of divisors:

$$
\psi_{1}=[\because 1 \cdot]+\frac{1}{12} \cdot \because:
$$

We must integrate Witten's class over each of these divisors. The first divisor contributes $0^{4}$ while the second one contributes

$$
\frac{1}{24} \sum_{a^{\prime}+a^{\prime \prime}=r-2}\left\langle\tau_{0, a_{1}} \ldots \tau_{0, a_{n}} \tau_{0, a^{\prime}} \tau_{0, a^{\prime \prime}}\right\rangle_{0}
$$

This is a combination of integrals of Witten's class (without $\psi$-classes) over genus 0 moduli spaces, and we are now going to determine its value. For simplicity, in this

\footnotetext{
${ }^{4}$ Indeed, the integral of $c_{W}$ over this divisor includes as a factor the integral of $c_{W}$ over a genus 1 moduli space, which vanishes for reasons of dimension: $\operatorname{deg} c_{W}=\left((r-2)(g-1)+\sum a_{i}\right) / r=\left(\sum a_{i}\right) / r \leq$ $n^{\prime}-1<n^{\prime}=\operatorname{dim} \overline{\mathcal{M}}_{1, n^{\prime}}$, where $n^{\prime}$ is the number of marked points and nodes on the torus.
} 
section we will use nonstandard notation for the bracket: $\left\langle a_{1}, \ldots, a_{n}\right\rangle$ instead of $\left\langle\tau_{0, a_{1}} \ldots \tau_{0, a_{n}}\right\rangle_{0}$.

Proposition 2.10 For any $m \leq r-2$ and for any $x_{1}, \ldots, x_{n}, 0 \leq x_{i} \leq r-2, \sum x_{i}=$ $n r-m-2$, we have

$$
\sum_{a+b=m}\left\langle a, b, x_{1}, \ldots, x_{n}\right\rangle=\frac{(n-1) !}{r^{n-1}} \prod_{i=1}^{n}\left(r-1-x_{i}\right) .
$$

Proof The proof uses the known initial values of the bracket:

$$
\begin{array}{rlrl}
\left\langle a_{1}, a_{2}, a_{3}\right\rangle & =1 & & \text { for } \sum a_{i}=r-2, \\
\left\langle a_{1}, a_{2}, a_{3}, a_{4}\right\rangle & =\frac{1}{r} \min _{1 \leq i \leq 4}\left(a_{i}, r-1-a_{i}\right) & \text { for } \sum a_{i}=2 r-2,
\end{array}
$$

and the WDVV equation. First note that the proposition is true for $n=1$ (it says that $\left.\sum_{a+b=m} 1=m+1\right)$.

Now we assume $n \geq 2$ and proceed by induction on $m$.

If $m=0$ then the only possible bracket is $\langle 0,0, r-2\rangle$.

Suppose that the proposition is true up to some $m$. Let us apply the WDVV equation to the correlators containing $1, a, b, x_{1}, \ldots, x_{n}$, the four distinguished points being 1 , $a, b$, and $x_{1}$. In other words, we consider all possible degenerations of the sphere into two components such that 1 and $a$ lie on one component, while $b$ and $x_{1}$ lie on the other one, and then we swap the four points. We obtain the following equality. (The summation over $a+b=m$ is implicitly assumed; a hat means that the symbol is skipped; underlined symbols are not in the original list, they appear at the node of the degenerate sphere in the WDVV formula.)

(2) $\langle 1, a, \underline{r-3-a}\rangle\left\langle\underline{a+1}, b, x_{1}, \ldots, x_{n}\right\rangle$

$$
\begin{aligned}
& +\sum_{i \neq 1}\left\langle 1, a, x_{i}, \underline{2 r-3-a-x_{i}}\right\rangle\left\langle\underline{a+1+x_{i}-r}, b, x_{1}, \ldots, \widehat{x_{i}}, \ldots, x_{n}\right\rangle \\
& =\left\langle 1, x_{1}, \underline{r-3-x_{1}}\right\rangle\left\langle a, b, \underline{x_{1}+1}, x_{2}, \ldots, x_{n}\right\rangle \\
& +\sum_{i \neq 1}\left\langle 1, x_{1}, x_{i}, \underline{2 r-3-x_{1}-x_{i}}\right\rangle \times \\
& \times\left\langle a, b, \underline{x_{1}+x_{i}+1-r}, x_{2}, \ldots, \widehat{x_{i}}, \ldots, x_{n}\right\rangle .
\end{aligned}
$$

The term (2) is the sum over $(a+1)+b=m+1$ that we want to determine. The missing case $a+1=0$ does not matter, because for $n \geq 2$ a single zero entry makes a bracket vanish. 
The term (3) can be evaluated by the induction assumption. It vanishes if $a+1+x_{i}<r$, while for $a+1+x_{i} \geq r$ we obtain a sum over $\left(a+1+x_{i}\right)+b=m+x_{i}-r<m$. Thus we have

$$
\text { (3) }=\frac{1}{r} \cdot \frac{(n-2) !}{r^{n-2}} \prod_{j \neq i}\left(r-1-x_{j}\right) .
$$

The term (4) can, once again, be evaluated by the induction assumption:

$$
\text { (4) }=\frac{(n-1) !}{r^{n-1}}\left(r-2-x_{1}\right) \prod_{j \neq 1}\left(r-1-x_{j}\right) \text {. }
$$

The last term (5) vanishes if $x_{1}+x_{i}+1<r$. Luckily, it turns out that for any $i>1$, we have $x_{1}+x_{i}+1 \geq r$. Indeed, if $a+b=m \leq r-3$ and $x_{1}+x_{j} \leq r-2$, then the sum of the $n-2$ remaining terms equals $n r-m-2-x_{1}-x_{i} \geq(n-2) r+3$, which is impossible since each of them equals at most $r-2$. Therefore we have

$$
(5)=\frac{1}{r} \cdot \frac{(n-2) !}{r^{n-2}}\left[\left(r-1-x_{1}\right)+\left(r-1-x_{i}\right)\right] \prod_{j \neq 1, i}\left(r-1-x_{j}\right) .
$$

We deduce that

$$
(2)=(4)+(5)-(3)=\frac{(n-1) !}{r^{n-1}} \prod_{j}\left(r-1-x_{j}\right) .
$$

Remark 2.11 By looking through the proof carefully one can check that the formula given in the proposition actually holds for $m \leq r$, except if $n=1$.

\section{Computations with double ramification cycles}

In this section we prove Theorem 2. By algebro-geometric arguments we find several relations binding the values of the brackets involved in the theorem, that is, the values of the integral of Witten's class $c_{W}\left(a_{1}, \ldots, a_{n}\right)$ over DR-cycles $D\left(k_{1}, \ldots, k_{n}\right) \subset \overline{\mathcal{M}}_{1, n}$. In Section 3.1 we write down these relations and prove that they suffice to determine the values of the bracket in all cases. We prove the relations in Section 3.2.

From now on and until the end of the section we assume that $\sum_{i=1}^{n} a_{i}=(n-1) r$. This is the maximal possible value, given that $0 \leq a_{i} \leq r-1$ and $r \mid \sum a_{i}$. Accordingly, the (complex) degree of $c_{W}\left(a_{1}, \ldots, a_{n}\right)$ equals $n-1$, and it is the highest possible value. In all other cases, the integrals we consider vanish for dimensional reasons. 


\subsection{The relations}

Denote by

$$
B=\left\langle\tau_{1, a_{1}} \tau_{0, a_{2}} \ldots, \tau_{0, a_{n}}\right\rangle_{1}=\frac{1}{24} \frac{(n-1) !}{r^{n-1}} \prod_{i=1}^{n}\left(r-1-a_{i}\right)
$$

(see Section 2.2).

Then the following relations hold.

\section{Relation 1}

$$
\begin{aligned}
& \left(k_{1}+1\right)\left(n_{+}+n_{-}+1\right) B=
\end{aligned}
$$

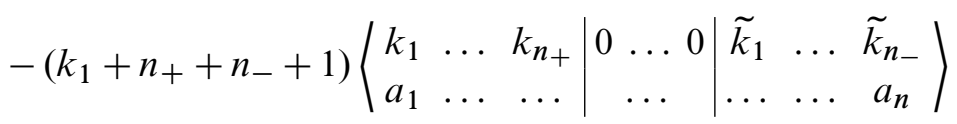

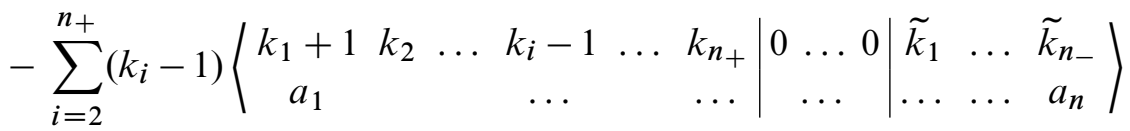

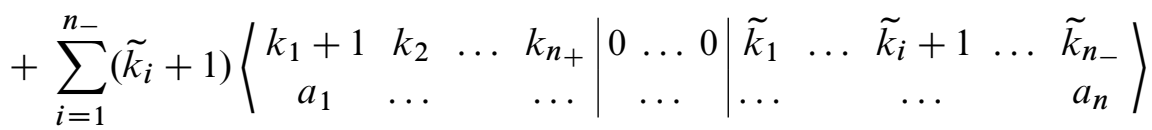

\section{Relation 2}

$$
\begin{aligned}
& \left(k_{1}+1\right) B=-\left\langle\begin{array}{ccc|c|ccc}
k_{1} & \ldots & k_{n_{+}} & \overbrace{0 \ldots 0}^{n_{0}} \ldots & \tilde{k}_{1} & \ldots & \tilde{k}_{n_{-}} \\
a_{1} & \ldots & \ldots & \ldots & \ldots & \ldots & a_{n}
\end{array}\right\rangle \\
& +\left\langle\begin{array}{cccc|c|c|ccc}
k_{1}+1 & k_{2} & \ldots & k_{n_{+}} & \overbrace{0}^{n_{0}-1} \ldots & 1 & \tilde{k}_{1} & \ldots & \tilde{k}_{n_{-}} \\
a_{1} & \ldots & \ldots & \ldots & \ldots & \ldots & \ldots & \ldots & a_{n}
\end{array}\right\rangle
\end{aligned}
$$

\section{Relation 3}

$$
\left\langle\begin{array}{c|cc|c}
1 & 0 & \ldots & 1 \\
a_{1} & \ldots & a_{n}
\end{array}\right|=0
$$

To these relations we may add the simple observation that the brackets are invariant under renumberings of the marked points and under a simultaneous change of sign of all $k_{i}$ 's.

One can check by direct computations that these relations are compatible with the expression of the bracket given in Theorem 2.

Lemma 3.1 Relations 1, 2, and 3 determine the values of all brackets unambiguously. 
Proof The lemma is proved by induction on the number of nonzero entries $n_{+}+n_{-}$ in a bracket, the base of induction being given by Relation 3 .

Consider a bracket whose value we would like to determine.

First case: suppose that one of the $\widetilde{k}_{i}$ 's is equal to 1 and one of the $k_{i}$ 's is greater than 1 (or vice versa). Then we take our bracket to be the last term in Relation 2 and replace it by the first term (plus a known multiple of $B$ ). We have decreased the number of nonzero $k_{i}$ 's in the bracket to be computed.

Second case: suppose that all the (nonzero) $k_{i}$ 's and $\tilde{k}_{i}$ 's equal 1 . In particular, $n_{+}=n_{-}$. If $n_{+}=n_{-}=1$ the bracket vanishes by Relation 3 . If $n_{+}=n_{-} \geq 2$, we take our bracket to be the first term in Relation 1. Our bracket is than replaced by a sum of brackets in which one of the $k_{i}$ 's and one of the $\tilde{k}_{i}$ 's are equal to 2 , while all the others are equal to 1 . Such brackets fall into the first case.

Third case: suppose that all of the $k_{i}$ 's and $\tilde{k_{i}}$ 's are greater than 1 . Then we take our bracket to be the first term of the second sum in Relation 1 and replace it by the sum of the other terms. As a result we obtain a combination of brackets in all of which

the number $\tilde{k}_{1}$ is smaller than in the initial bracket. Repeating the same operation for each bracket we are sure that $\tilde{k}_{1}$ will decrease by 1 with every step. Thus after a finite number of steps we will end up with a collection of brackets all of which fall into the first two cases.

Thus the relations determine the values of the brackets unambiguously. Since they are compatible with the expression of Theorem 2, the theorem will be completely proved once we will have proved the relations.

\subsection{Proof of the relations}

First of all, Relation 3 is obvious. The bracket in this relation denotes an integral over an empty DR-cycle $\bar{D}(1,0, \ldots, 0,-1)$, therefore it vanishes. Thus we only need to prove Relations 1 and 2.

Consider the commutative diagram involving four moduli spaces shown in Figure 2.

Here $\overline{\mathbf{A}}=\overline{\mathbf{A}}\left(k_{1}+1, \ldots, k_{n},-1\right)$ is the DR-space of genus 1 admissible coverings with ramification types $\left(k_{1}+1, k_{2}, \ldots, k_{n_{+}}\right)$and $\left(1, \tilde{k}_{1}, \ldots, \tilde{k}_{n_{-}}\right)$over 0 and $\infty$. There are also $n_{0}$ additional marked points on the image curve $S$ and, accordingly, $n_{0}$ black round marked points on the source curve $C$.

Recall that the map $h: \overline{\mathbf{A}} \rightarrow \overline{\mathcal{M}}_{1, n}$ forgets all marked points on $C$ except the round black dots and stabilizes the curve. 

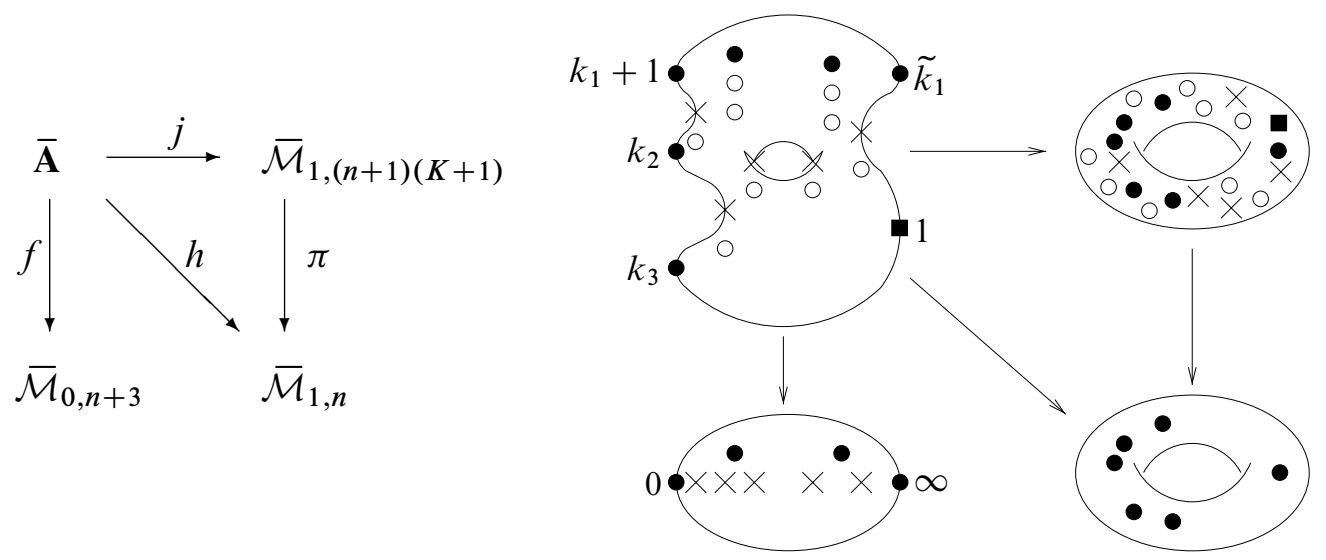

Figure 2: Four moduli spaces

In this example, the DR-cycle coincides with the whole moduli space $\overline{\mathcal{M}}_{1, n}$.

The main technique of this section is to compute the integral

$$
I=\int_{\overline{\mathbf{A}}} h^{*}\left(c_{W}\right) \psi_{1}
$$

by three different methods and to use the relations thus obtained. The three methods can be summarized as follows.

(A) Express the $\psi$-class on $\overline{\mathcal{M}}_{0, n+3}$ as a sum of boundary divisors. By Ionel's lemma (Lemma 2.3), the preimages under $f$ of these boundary divisors represent the class $\psi_{1}$ on $\overline{\mathbf{A}}$. The images of these divisors under $h$ turn out to be DRcycles. Therefore the integrals of Witten's class over these divisors are some values of the bracket of Definition 1.1.

(B) Same as (A) with another expression of the $\psi$-class in terms of boundary divisors.

(C) In Section 2.2 we computed the integral

$$
B=\int_{\overline{\mathcal{M}}_{1, n}} c_{W} \psi_{1}
$$

analogous to $I$. Now, the classes $\psi_{1}$ on $\overline{\mathcal{M}}_{1, n}$ and on $\overline{\mathbf{A}}$ differ by a sum of boundary divisors. The images of these divisors under $h$ turn out, once again, to be DR-cycles, and integrating Witten's class upon them we obtain a linear combination of brackets. 
(A) On the space $\overline{\mathcal{M}}_{0, n+3}$ in Figure 2, consider the class $\psi$ at the point labeled by 0 . This class is equal to a sum of boundary divisors that we will represent as:

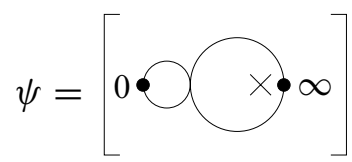

The picture represents a sum of boundary divisors where the sphere splits into two components. The point labeled by 0 is on the first component, while the point labeled by $\infty$ together with some chosen branch point (say, the first one) is on the second component. The other marked points on the sphere can be distributed arbitrarily between the components.

Consider the preimages under $f$ of these boundary strata.

Lemma 3.2 Among the preimages in $\overline{\mathbf{A}}$ of the divisors (6) consider those on which the integral of $h^{*}\left(c_{W}\right)$ does not vanish. These divisors are $\operatorname{DIV}_{1}, \operatorname{DIV}_{i}$, and $\widetilde{\mathrm{DIV}}_{i}$ in Figure 3.

It should be understood that each picture actually represents a generic admissible covering lying in the divisor. In other words, the divisor is the closure of the set of admissible coverings with the topological structure shown in Figure 3.
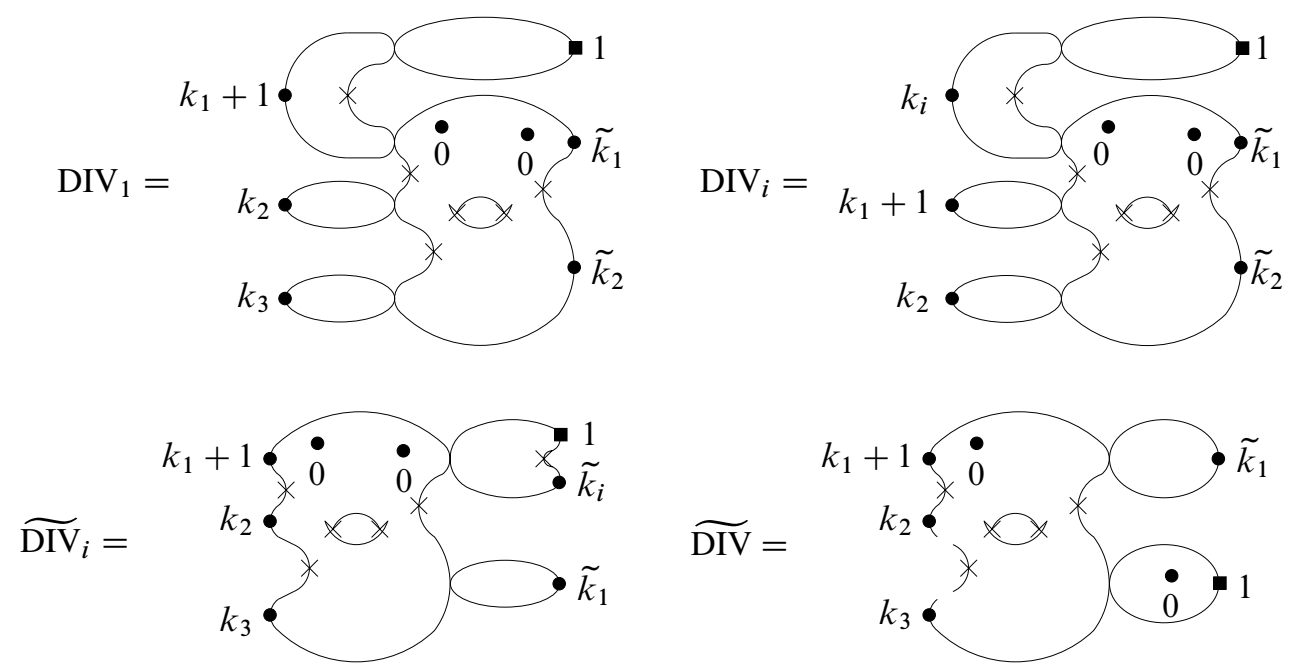

Figure 3: Divisors in $\overline{\mathbf{A}}$ 
Proof of Lemma 3.2 We consider an irreducible component of the preimage of (6) and reason in terms of the generic admissible covering $\varphi: C \rightarrow S$ in this component.

First note that the curve $C$ cannot contain a pinched sphere (a rational irreducible component with two identified points). Indeed, every node in $C$ necessarily separates two different components: one containing preimages of 0 from one containing preimages of $\infty$.

Suppose that $C$ does not have a toric component. Then it looks as a ring of spheres with several "tails":

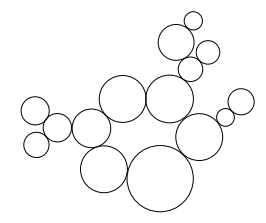

Each sphere of the ring contains at least 2 nodes. Thus the restriction of $\varphi$ to any such sphere is of degree at least 2. Which implies that there is at least one round black dot on each sphere of the ring (there is at least one preimage of 0 or $\infty$ on each component, and the square black dot alone, being a simple preimage, is not enough for $\varphi$ to have degree 2). It follows that none of the spheres of the ring is contracted under the map $h$ to $\overline{\mathcal{M}}_{1, n}$. Now, the number of spheres in the ring is even (those that contain a preimage of 0 alternate with those that contain a preimage of $\infty$ ). Thus the curve $C$ retains at least 2 nodes after the projection to $\overline{\mathcal{M}}_{1, n}$. Which means that the codimension of the image of the corresponding divisor under $h$ is at least 2, so the integral of Witten's class on it vanishes. The conclusion is that we only need to consider curves $C$ with a toric component.

Suppose $C$ has a toric component with several "tails":

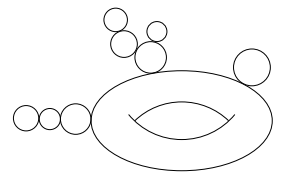

If one of the tails contains more than one round black dot, than the projection $h$ of such a curve to $\overline{\mathcal{M}}_{1, n}$ looks like:

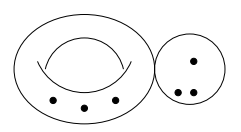


The integral of Witten's class over the divisor of such curves vanishes (see Footnote 4). The conclusion is that each "tail" contains at most one round black dot.

Note that every component of $C$ contains at least one black dot (round or square): either a preimage of 0 or of $\infty$. It follows that each tail (except perhaps one) is composed of exactly 1 sphere containing exactly 1 black dot. The exceptional tail can be composed of 1 or 2 spheres containing a round black dot and a square black dot. On each simple tail the function $\varphi$ has the form $z \mapsto z^{k}$ for some positive integer $k$.

Actually, an exceptional tail is bound to exist. Indeed, suppose there is no exceptional tail, ie, every tail is composed of 1 sphere with a unique black dot. The image curve $S$ has 2 components, and in the case we consider, the preimage of one of them is the toric component of $C$, while the preimage of the other is the union of the tails. Then the latter component of $S$ contains no marked points except 0 or $\infty$. This is impossible, because $S$ is stable.

If the exceptional tail is composed of 1 sphere, then this sphere contains the black square dot and some dot $\tilde{k}_{i}$. This gives the divisor $\widetilde{\mathrm{DIV}}_{i}$. If the exceptional tail is composed of 2 spheres, then one of them contains the square black dot, and the other one some dot $k_{i}$ or $k_{1}+1$. The sphere with the square dot can contain at most one node because the degree of $\varphi$ on it equals 1 . Thus we obtain the divisors DIV $_{1}$ and $\mathrm{DIV}_{i}$.

Lemma 3.2 is proved.

Now, the integral $I$ is the sum of integrals of Witten's class over the divisors DIV , $_{1}$ $\mathrm{DIV}_{i}$, and $\widetilde{\mathrm{DIV}}_{i}$, which we will now determine.

Lemma 3.3 The contributions to $I$ of the divisors $\mathrm{DIV}_{1}, \mathrm{DIV}_{i}$, and $\widetilde{\mathrm{DIV}}_{i}$ equal

$$
\begin{aligned}
& \frac{\left(n_{+}+n_{-}\right) !(K-1) !^{n_{+}+n_{-}+1} K !^{n_{0}}}{k_{1}+1} \cdot k_{1}\left(n_{+}+n_{-}\right) \times
\end{aligned}
$$

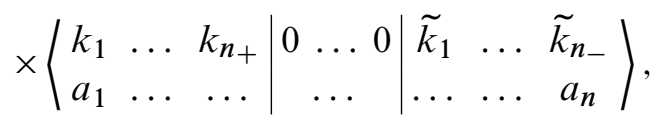

$$
\begin{aligned}
& \frac{\left(n_{+}+n_{-}\right) !(K-1) !^{n_{+}+n_{-}+1} K !^{n_{0}}}{k_{1}+1} \cdot\left(k_{i}-1\right)\left(n_{+}+n_{-}\right) \times
\end{aligned}
$$

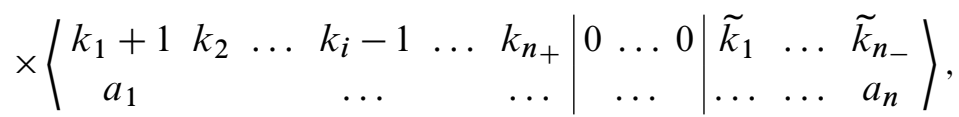




$$
\begin{aligned}
& \frac{\left(n_{+}+n_{-}\right) !(K-1) !^{n_{+}+n_{-}+1} K !^{n_{0}}}{k_{1}+1} \cdot\left(\tilde{k}_{i}+1\right) \times
\end{aligned}
$$

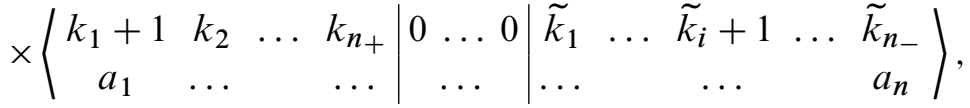

respectively, where $K=\sum k_{i}=\sum \tilde{k}_{i}$.

Proof The fundamental classes of the divisors $\mathrm{DIV}_{1}, \mathrm{DIV}_{i}$, and $\widetilde{\mathrm{DIV}}_{i}$ project to multiples of double ramification divisors in $\overline{\mathcal{M}}_{1, n}$ under the map $h$. Thus we can integrate Witten's class over these double ramification divisors, which explains the brackets that appear in the answers.

The coefficients in front of these brackets arise as products of three following factors.

(i) The transversal multiplicity of the map $f$ on the divisor. Consider a generic admissible covering $\varphi: C \rightarrow S$ in one of the divisors. Its image $S \in \overline{\mathcal{M}}_{0, n+3}$ under the map $f$ has exactly one node. Consider a smooth curve $\widetilde{S}$ close to $S$ in $\overline{\mathcal{M}}_{0, n+3}$. Then $\widetilde{S}$ will have several preimages in $\overline{\mathbf{A}}$ close to $\varphi$. The number of such preimages is called the transversal multiplicity of $f$ on the divisor. It is readily seen to be equal to the product of indices of all nodes of $C$, because every node can be smoothed in the number of ways equal to its index, as $S$ goes to $\tilde{S}$. Since we need pullbacks under $f$ of homology classes rather than geometric preimages, we must take every divisor in $\overline{\mathbf{A}}$ with weight equal to the corresponding transversal multiplicity.

(ii) The degree of $h$ on the divisor. This is simply the number of ways to label the marked points of $C$ that are forgotten by $h$.

(iii) The factor $1 /\left(k_{1}+1\right)$ coming from Ionel's lemma.

For $\mathrm{DIV}_{i}$, these factors are:

$$
\text { (i) }=\left(k_{1}+1\right) k_{2} \ldots k_{i-1}\left(k_{i}-1\right) k_{i+1} \ldots k_{n_{+}} \text {. }
$$

This is the product of the indices of all nodes of $C$.

$$
\text { (ii) }=\frac{\left(n_{+}+n_{-}\right)\left(n_{+}+n_{-}\right) !(K-1) !^{n_{+}+n_{-}+1} K !^{n_{0}}}{\left(k_{1}+1\right) k_{2} \ldots k_{i-1} k_{i+1} \ldots k_{n_{+}}} \text {. }
$$

The factor $\left(n_{+}+n_{-}\right)\left(n_{+}+n_{-}\right)$! is the number of ways to number the branch points on the two-component genus 0 image curve, taking into account that the first ramification point lies on the component of $\infty$. $(K-1) !^{n_{+}+n_{-}+1}$ is the number of ways to number the white (noncritical) preimages of the ramification points. $K !^{n_{0}}$ is the number of 
ways to number the white preimages of the black marked points different from 0 and $\infty$. The denominator comes from the spheres on the left of the picture of $\operatorname{DIV}_{i}$ : the restriction of the admissible covering map $\varphi$ on these spheres has the form $z^{k}$, and thus $z \mapsto e^{2 \pi i / k} z$ gives a renumbering of the white dots on such a sphere equivalent to the initial numbering.

Multiplying these factors (without forgetting $1 /\left(k_{1}+1\right)$ ) we obtain the coefficient for $\mathrm{DIV}_{i}$ as claimed in the lemma. The computations for the other divisors are analogous, so we only give the answers.

For DIV $_{1}$ the factors (i) and (ii) are

$$
\begin{aligned}
\text { (i) } & =k_{1} k_{2} \ldots k_{n_{+}} \\
\text {(ii) } & =\frac{\left(n_{+}+n_{-}\right)\left(n_{+}+n_{-}\right) !(K-1) !^{n_{+}+n_{-}+1} K !^{n_{0}}}{k_{2} \ldots k_{n_{+}}} .
\end{aligned}
$$

For $\widetilde{\mathrm{DIV}}_{i}$ the factors (i) and (ii) are

$$
\begin{aligned}
\text { (i) } & =\tilde{k}_{1} \ldots \tilde{k}_{i-1}\left(\tilde{k}_{i}+1\right) \tilde{k}_{i+1} \ldots \tilde{k}_{n_{-}} . \\
\text {(ii) } & =\frac{\left(n_{+}+n_{-}\right) !(K-1) !^{n_{+}+n_{-}+1} K !^{n_{0}}}{\tilde{k}_{1} \ldots \tilde{k}_{i-1} \tilde{k}_{i+1} \ldots \tilde{k}_{n_{-}}} .
\end{aligned}
$$

(B) The second way of computing the integral $I$ is not very different from the first one. This time we start with a different presentation of the $\psi$-class at the point labeled by 0 on $\overline{\mathcal{M}}_{0, n+3}$ :

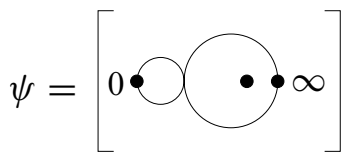

The picture represents the sum of boundary divisors where the sphere splits into two components: the point labeled by 0 is on the first component, while the point labeled by $\infty$ together with another chosen black marked point (say, the first one) is on the second component.

Lemma 3.4 Among the preimages in $\overline{\mathbf{A}}$ of the divisors (7) consider those on which the integral of $c_{W}$ does not vanish. These divisors are $\mathrm{DIV}_{1}, \mathrm{DIV}_{i}$, and $\widetilde{\mathrm{DIV}}$ in Figure 3.

Proof First suppose there are no points marked with crosses on the component of $\infty$ on the image curve. This means that the preimages of this component are spheres containing only black marked points. However, all these components must be contracted under the map $h$. (Otherwise the integral of $c_{W}$ over this divisor vanishes; 
see Footnote 4.) Therefore, actually, every sphere contains exactly one black point, except one sphere that contains a round black dot and a square black dot. This is the divisor $\widetilde{\mathrm{DIV}}$.

If the component of $\infty$ in the image curve contains at least one cross-marked point, then we have reduced the problem to the situation of Lemma 3.2. Thus the only divisors that can give a nonzero contribution are $\operatorname{DIV}_{1}, \operatorname{DIV}_{i}$, and $\widetilde{\mathrm{DIV}}_{i}$. However, actually the divisors $\widetilde{\mathrm{DIV}}_{i}$ do not appear as preimages of the divisors (7) because in this case the component of $\infty$ in the image contains no black dots other than $\infty$.

Lemma 3.5 The contributions to $I$ of the divisors $\operatorname{DIV}_{1}, \operatorname{DIV}_{i}$, and $\widetilde{\mathrm{DIV}}$ equal

$$
\begin{aligned}
& \frac{\left(n_{+}+n_{-}\right) !(K-1) !^{n_{+}+n_{-}+1} K !^{n_{0}}}{k_{1}+1} \cdot k_{1}\left(n_{+}+n_{-}+1\right) \times
\end{aligned}
$$

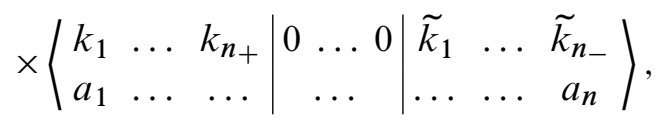

$$
\begin{aligned}
& \frac{\left(n_{+}+n_{-}\right) !(K-1) !^{n_{+}+n_{-}+1} K !^{n_{0}}}{k_{1}+1} \cdot\left(k_{i}-1\right)\left(n_{+}+n_{-}+1\right) \times
\end{aligned}
$$

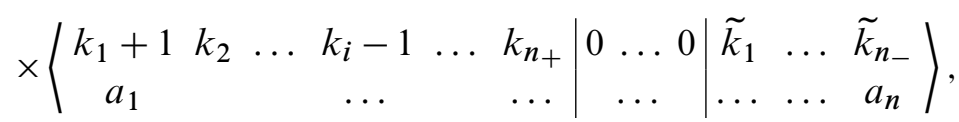

$$
\begin{aligned}
& \frac{\left(n_{+}+n_{-}\right) !(K-1) !^{n_{+}+n_{-}+1} K !^{n_{0}}}{k_{1}+1} \cdot\left(n_{+}+n_{-}+1\right) \times
\end{aligned}
$$

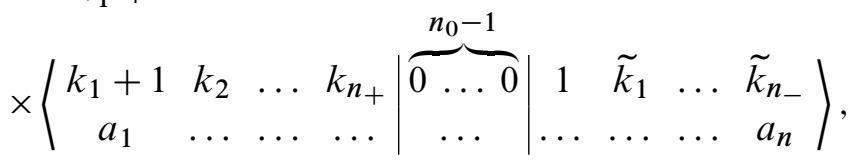

respectively, where $K=\sum k_{i}=\sum \tilde{k_{i}}$.

Proof The proof is analogous to that of Lemma 3.3: every coefficient is the product of three factors:

(i) the transversal multiplicity of $f$ on the divisor, or, in other words, the product of the node indices of the generic curve $C$;

(ii) the degree of $h$ on the divisor; and

(iii) the factor $1 /\left(k_{1}+1\right)$ coming from Ionel's lemma.

The computation of the degree of $h$ is slightly different from that in Lemma 3.3. Indeed, in Lemma 3.3 the first step was to label the $n_{+}+n_{-}+1$ branch points on $S$, under the 
restriction that the first of them lies on the component of $\infty$. In the present situation, we count all the ways to label the marked points, with no restrictions. At first sight it may seem that we do have a restriction: the first of the $n_{0}$ additional marked points must lie on the component of $\infty$. However, actually, the $n_{0}$ additional marked points are not forgotten by $h$; so they are already labeled and this condition is already satisfied.

For $\mathrm{DIV}_{1}$ we have

$$
\begin{aligned}
\text { (i) } & =k_{1} k_{2} \ldots k_{n_{+}} \\
\text {(ii) } & =\frac{\left(n_{+}+n_{-}+1\right) !(K-1) !^{n_{+}+n_{-}+1} K !^{n_{0}}}{k_{2} \ldots k_{n_{+}}} .
\end{aligned}
$$

For $\mathrm{DIV}_{i}$ we have

$$
\begin{aligned}
\text { (i) } & =\left(k_{1}+1\right) k_{2} \ldots k_{i-1}\left(k_{i}-1\right) k_{i+1} \ldots k_{n_{+}} \\
\text {(ii) } & =\frac{\left(n_{+}+n_{-}+1\right) !(K-1) !^{n_{+}+n_{-}+1} K !^{n_{0}}}{\left(k_{1}+1\right) k_{2} \ldots k_{i-1} k_{i+1} \ldots k_{n_{+}}} .
\end{aligned}
$$

For $\widetilde{\mathrm{DIV}}$ we have

$$
\begin{aligned}
\text { (i) } & =\tilde{k}_{1} \ldots \tilde{k}_{n_{-}} . \\
\text {(ii) } & =\frac{\left(n_{+}+n_{-}+1\right) !(K-1) !^{n_{+}+n_{-}+1} K !^{n_{0}}}{\tilde{k}_{1} \ldots \tilde{k}_{n_{-}}} .
\end{aligned}
$$

(C) Now we are going to evaluate

$$
I=\int_{\overline{\mathbf{A}}} h^{*}\left(c_{W}\right) \psi_{1}(\overline{\mathbf{A}})
$$

using the value

$$
B=\int_{\overline{\mathcal{M}}_{1, n}} c_{W} \psi_{1}\left(\overline{\mathcal{M}}_{1, n}\right)
$$

If the class $\psi_{1}$ on $\overline{\mathbf{A}}$ were a pullback of the class $\psi_{1}$ on $\overline{\mathcal{M}}_{1, n}$, then we would simply have $I=B \cdot \operatorname{deg} h$. However this equality is actually incorrect, because $h^{*}\left(\psi_{1}\left(\overline{\mathcal{M}}_{1, n}\right)\right)$ and $\psi_{1}(\overline{\mathbf{A}})$ differ.

Lemma 3.6 The difference $\psi_{1}(\overline{\mathbf{A}})-h^{*}\left(\psi_{1}\left(\overline{\mathcal{M}}_{1, n}\right)\right)$ can be represented as the sum of divisors

$$
k_{2} \ldots k_{n_{+}} \mathrm{DIV}_{1}+\sum_{i=2}^{n_{+}} k_{2} \ldots k_{i-1}\left(k_{i}-1\right) k_{i+1} \ldots k_{n_{+}} \mathrm{DIV}_{i}
$$


(the divisors being shown in Figure 3) and, in addition, some other divisors on which the integral of $c_{W}$ vanishes.

Proof There is a subtlety in finding the difference $\psi_{1}(\overline{\mathbf{A}})-h^{*}\left(\psi_{1}\left(\overline{\mathcal{M}}_{1, n}\right)\right)$. First consider the difference $\psi_{1}\left(\overline{\mathcal{M}}_{1,(n+1)(K+1)}\right)-\pi^{*}\left(\psi_{1}\left(\overline{\mathcal{M}}_{1, n}\right)\right)$ in the upper right moduli space in Figure 2. It is given by the divisor $D_{1}$ of all stable curves on which the first black marked point $\left(k_{1}+1\right)$ is situated on a component contracted by $\pi$. Now we consider the intersection of this divisor with the image $j(\overline{\mathbf{A}})$. It turns out that this intersection is not necessarily transversal, and its multiplicity gives the coefficients that appear in the formulation of the lemma.

Now we take the pullback of the intersection by $j$. Let us consider an irreducible component of this pullback

$$
j^{*}\left(\psi_{1}\left(\overline{\mathcal{M}}_{1,(n+1)(K+1)}\right)-\pi^{*}\left(\psi_{1}\left(\overline{\mathcal{M}}_{1, n}\right)\right)\right)
$$

and a generic admissible covering $\varphi$ in this component.

The image curve of $\varphi$ in $\overline{\mathcal{M}}_{0, n+3}$ has a unique node (otherwise the codimension of such a component in $\overline{\mathbf{A}}$ would be at least 2).

Moreover, this node separates 0 and $\infty$. Indeed, otherwise the component containing the point $k_{1}+1$ also contains some preimage of $\infty$. Since this component is contracted by $h$, the only possible preimage is the square black dot (and there are no other preimages of $\infty$ ). Thus the degree of $\varphi$ on this component equals 1 . But this implies $k_{1}+1=1$, which is impossible.

On the component of $\infty$ there exists at least one cross marked point. Indeed, otherwise there is no ramification over this component, so all the ramification is over the component containing 0 . Then the point $k_{1}+1$ lies on the torical component, which is impossible, since this component should be contracted by $h$.

Thus we have reduced the problem to the situation of Lemma 3.2 with the additional restriction that the component containing the point $k_{1}+1$ should be contracted by $h$. This restriction excludes the divisors $\widetilde{\mathrm{DIV}}_{i}$ but allows the divisors $\mathrm{DIV}_{1}$ and $\mathrm{DIV}_{i}$.

It remains to find the multiplicity of the intersection $j(\overline{\mathbf{A}}) \cap D_{1}$ along $j\left(\operatorname{DIV}_{1}\right)$ and $j\left(\mathrm{DIV}_{i}\right)$. This multiplicity is easily seen to be equal to the product of indices of the nodes that are smoothed as a generic point of the intersection moves to a generic point of $D_{1}$ (that is, all nodes except one). Such products of indices are precisely the coefficients stated in the lemma. 
Lemma 3.7 The contributions to $I$ of the integral $B$ and that of the divisors DIV $_{1}$ and $\mathrm{DIV}_{i}$ equal

$$
\begin{aligned}
& \frac{\left(n_{+}+n_{-}\right) !(K-1) !^{n_{+}+n_{-}+1} K !^{n_{0}}}{k_{1}+1} \cdot\left(k_{1}+1\right)\left(n_{+}+n_{-}+1\right) \cdot B, \\
& \frac{\left(n_{+}+n_{-}\right) !(K-1) !^{n_{+}+n_{-}+1} K !^{n_{0}}}{k_{1}+1} \cdot\left(k_{1}+1\right)\left(n_{+}+n_{-}+1\right) \times \\
& \times\left(\begin{array}{ccc|c|c|ccc}
k_{1} & \ldots & k_{n_{+}} & 0 & \ldots & \tilde{k}_{1} & \ldots & \tilde{k}_{n_{-}} \\
a_{1} & \ldots & \ldots & \ldots & \ldots & \ldots & a_{n}
\end{array}\right\rangle, \\
& \frac{\left(n_{+}+n_{-}\right) !(K-1) !^{n_{+}+n_{-}+1} K !^{n_{0}}}{k_{1}+1} \cdot\left(k_{i}-1\right)\left(n_{+}+n_{-}+1\right) \times
\end{aligned}
$$

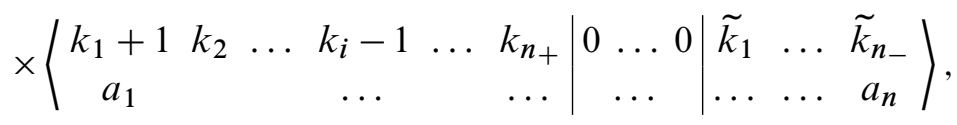

respectively, where $K=\sum k_{i}=\sum \tilde{k}_{i}$.

Proof The coefficient of $B$ is just the degree of $h$. The coefficients of the other two brackets are obtained as products of two factors:

(i) the coefficients of the divisors appearing in Lemma 3.6 and

(ii) the degree of $h$ on the divisor.

Here, as in Lemma 3.5, when we compute the degree of $h$ we count all possible ways to label the marked points on $C$ with no restriction.

For DIV $_{1}$ these factors equal:

$$
\begin{aligned}
\text { (i) } & =k_{2} \ldots k_{n_{+}} \cdot \\
\text { (ii) } & =\frac{\left(n_{+}+n_{-}+1\right) !(K-1) !^{n_{+}+n_{-}+1} K !^{n_{0}}}{k_{2} \ldots k_{n_{+}}} .
\end{aligned}
$$

For $\mathrm{DIV}_{i}$ these factors equal:

$$
\begin{aligned}
& \text { (i) } k_{2} \ldots k_{i-1}\left(k_{i}-1\right) k_{i+1} \ldots k_{n_{+}} \\
& \text {(ii) } \frac{\left(n_{+}+n_{-}+1\right) !(K-1) !^{n_{+}+n_{-}+1} K !^{n_{0}}}{\left(k_{1}+1\right) k_{2} \ldots k_{i-1} k_{i+1} \ldots k_{n_{+}}} .
\end{aligned}
$$


Thus we have established three expressions, (A), (B), and (C) for the integral $I$.

Writing $(\mathrm{A})=(\mathrm{C})$ we obtain Relation 1: the first term of Lemma 3.7 gives the lefthand side, while the other terms of Lemma 3.7 and Lemma 3.3 combine to give the right-hand side.

Similarly, the equality $(\mathrm{B})=(\mathrm{C})$ gives Relation 2 .

\section{An algorithm to compute Witten's intersection numbers}

Here we present an algorithm for computing any number $\left\langle\tau_{d_{1}, a_{1}} \ldots \tau_{d_{n}, a_{n}}\right\rangle$. This algorithm is rather hard to implement on a computer because it involves enumerating all possible degenerations of an admissible covering satisfying some given properties. Thus this section is best viewed as a constructive proof of Theorem 1. Examples of concrete application of steps of this algorithm can be found in [18].

Suppose we are given $n$ nonnegative integers $d_{1}, \ldots, d_{n}$. Choose an integer $p, 0 \leq$ $p \leq g$ and $n$ numbers $k_{1}, \ldots, k_{n}$ with sum $\sum k_{i}=p$ and bounded by $\left|k_{i}\right|>g \sum_{j} d_{j}$. Consider the DR-space $\overline{\mathbf{A}}=\overline{\mathbf{A}}\left(k_{1}, \ldots, k_{n},-1, \ldots,-1\right)$, where the list ends with $p$ numbers -1 so that the total sum is 0 as it should be.

As explained in Section 2.1, the forgetful map $h: \overline{\mathbf{A}} \rightarrow \overline{\mathcal{M}}_{g, n}$ sends $\overline{\mathbf{A}}$ to the DR-cycle $\bar{D}_{g, n}\left(k_{1}, \ldots, k_{n},-1, \ldots,-1\right)$ of codimension $g-p$ in $\overline{\mathcal{M}}_{g, n}$.

We are going to compute the integral

$$
\int_{\overline{\mathbf{A}}} h^{*}\left(c_{W} \psi_{1}^{d_{1}} \ldots \psi_{n}^{d_{n}}\right)=\operatorname{deg}(h) \int_{\bar{D}} c_{W} \psi_{1}^{d_{1}} \ldots \psi_{n}^{d_{n}} .
$$

If $p=g$ this will give us the value of the bracket $\left\langle\tau_{d_{1}, a_{1}} \ldots \tau_{d_{n}, a_{n}}\right\rangle$. Note, however that our result is actually more general.

Our algorithm for computing Integral (8) can be summed up as follows.

While the class $\psi_{i}$ on $\overline{\mathcal{M}}_{g, n}$ is, in general, not representable by boundary divisors, its pullback to $\overline{\mathbf{A}}$ can be easily represented as a sum like that. At each step we will consider one $\psi$-class and replace it by a sum of divisors. Thus we will be reduced to computing the integral of a product $c_{W} \cdot$ (powers of $\psi$-classes) over some divisors, the number of $\psi$-classes having decreased by 1 . Using the factorization rules for Witten's class, we will be able to represent each integral over a boundary divisor as a product of analogous integrals over simpler DR-spaces.

Proposition 4.1 The integral (8) can be effectively expressed as a combination of analogous integrals with a smaller sum $\sum d_{i}$. 
Proof (1) Expressing the pullback $h^{*}\left(\psi_{i}\right)$ as a sum of boundary divisors of $\overline{\mathbf{A}}$.

Consider the class $\psi_{i}=\psi_{i}\left(\overline{\mathcal{M}}_{g, n}\right)$ on $\overline{\mathcal{M}}_{g, n}$ and the corresponding class $\Psi_{i}=\psi_{i}(\overline{\mathbf{A}})$ on $\overline{\mathbf{A}}$. The pullback $h^{*}\left(\psi_{i}\right)$ to $\overline{\mathbf{A}}$ can be represented as a sum of divisors in the following way. First, the class $\Psi_{i}$ can, by Ionel's lemma (Lemma 2.3), be replaced by the pullback of the $\psi$-class at 0 or at $\infty$ on $\overline{\mathcal{M}}_{0, n+p+2 g}$. The latter class is easy to represent by a sum of divisors; see Equation (6). Second, the difference $\Psi_{i}-h^{*}\left(\psi_{i}\right)$ is equal to the sum (with certain coefficients) of divisors $D_{i}$ formed by the admissible coverings for which the component of the source curve containing the $i$-th marked point is contracted by $h$ (cf Lemma 3.6).

(2) The image curve of a generic admissible covering in every boundary divisor has a unique node separating 0 and $\infty$.

Let us consider an irreducible component of one of the above divisors and a generic admissible covering $\varphi$ in this component. The image curve of $\varphi$ in $\overline{\mathcal{M}}_{0, n+p+2 g}$ has a unique node because otherwise the codimension of such a component in $\overline{\mathbf{A}}$ would be at least 2 .

Let us prove that this node separates 0 and $\infty$. Indeed, this is obvious by construction for the divisors involved in the expression of $\Psi_{i}$. As for the divisors representing the difference $\Psi_{i}-h^{*}\left(\psi_{i}\right)$, suppose that the node on the image curve does not separate 0 and $\infty$. Consider the component of the source curve $C$ containing the $i$-th black round marked point. This component must contain both a preimage of 0 and a preimage of $\infty$. On the other hand, it is, by construction, contracted by $h$. Thus the only possibility is that it contains a unique round black dot (a preimage of 0 ) and one or several square black dots (since these are forgotten by $h$ ). But then the degree of $\varphi$ on this component is equal to $\left|k_{i}\right|$ and, at the same time, to the number of square black dots. Since we assumed that $\left|k_{i}\right|>g \sum d_{j} \geq g \geq p$, this is impossible.

(3) Splitting the admissible covering.

Denote by DIV the boundary divisor of $\overline{\mathbf{A}}$ under consideration.

Our aim is to construct a map

$$
u: \mathrm{DIV} \rightarrow \overline{\mathbf{A}}_{1} \times \cdots \times \overline{\mathbf{A}}_{c}
$$

from DIV to a product of DR-spaces. This is done as follows.

Take an admissible covering $\varphi: C \rightarrow S$ from DIV. For a generic $f$, the curve $S$ has exactly one node: we call this node essential. For a nongeneric $f$, the curve $S$ can have other nodes in addition to the essential one. Similarly, call essential nodes of $C$ 
the preimages of the essential node of $S$. These nodes are present for all admissible coverings of $f$, but for a nongeneric admissible covering, $C$ can have more nodes.

Now split (in other words, normalize) the essential nodes of $C$ and of $S$. The curve $S$ splits into two rational curves, 0 lying on one component and $\infty$ on the other. The curve $C$ splits in several connected components that we call parts. This is shown in Figure 4 (the meaning of various markings will be explained later). The parts are denoted by roman numerals.

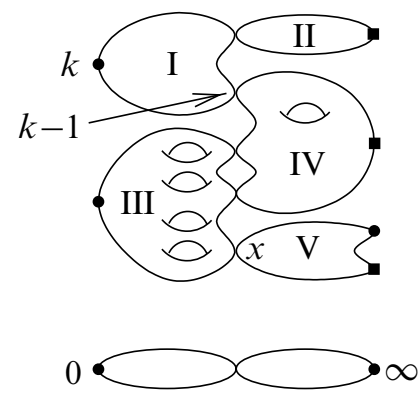

Figure 4: A boundary divisor is isomorphic to a product of several simpler DR-spaces.

Thus for each part $j$ of $C$ we have obtained a map $\varphi_{j}: C_{j} \rightarrow S_{j}$. (The curves $C_{j}$ are the parts of $C$ and hence different for different $j$ 's, while $S_{j}$ is one of the two components of $S$ and hence can be the same for different $j$ 's.)

The map $\varphi_{j}$ is not necessarily an admissible covering. Indeed, in most cases there is a black round dot or a cross on $S_{j}$ that has only white round dots as preimages in the part $C_{j}$. Consider, for instance, a critical point (a cross) on part IV in Figure 4. Its image is a branch point (a cross) in $S$. This cross has several only (white) preimages on parts II and V.

Once we have separated the parts, we must forget these "useless" white points. More precisely, we (i) forget those marked points on $S_{j}$ that have only white preimages in $C_{j}$ (together with these white preimages), (ii) contract the unstable components of $S_{j}$ and their preimages in $C_{j}$. If, once we have forgotten all the useless marked points, the curve $S_{j}$ has become unstable, then we ignore the corresponding part altogether. We say that this part has vanished. For example, in Figure 4 part II vanishes.

Denote by $c$ the number of parts that have not vanished after this procedure ( $c=4$ in our example). Denote by $\overline{\mathbf{A}}_{1}, \ldots, \overline{\mathbf{A}}_{c}$ the corresponding DR-spaces. We have constructed a map

$$
u: \mathrm{DIV} \rightarrow \overline{\mathbf{A}}_{1} \times \cdots \times \overline{\mathbf{A}}_{c} .
$$


We have $\operatorname{dim}$ DIV $\geq \sum \operatorname{dim} \overline{\mathbf{A}}_{j}$. The inequality becomes an equality if and only if $c=2$. In other words: above each of the two components of $S$, all parts except one contain only white dots and hence vanish ${ }^{5}$.

When the dimensions coincide, $u$ sends the fundamental homology class of DIV to a multiple of the fundamental homology class of the product in the image. Indeed, $u$ is a nonramified covering on the open part of DIV where the curve $S$ has exactly one node.

(4) Re-assigning the $\psi$-classes.

Recall that the map $h$ takes the source curve in Figure 4, forgets all the marked points except the round black dots and stabilizes the curve. We are interested in the preimages of the $\psi$-classes under $h$. During the stabilization the marked points from certain parts can land on some other parts. In the figure, the $\psi$-class assigned to the point labeled with $k$ (on part I) will land on part IV. Similarly, the $\psi$-class assigned to the round black dot of part V will land on part III, at the point marked with an $x$.

Lemma 4.2 Every class $h^{*}\left(\psi_{i}\right)$ on DIV coincides with the class $h_{j}^{*}\left(\psi_{l}\right)$ on $\overline{\mathbf{A}}_{j}$ for some $j$ and $l$.

Proof Stabilizing the source curve is equivalent to first contracting the unstable parts, then stabilizing the curve thus obtained. The class $h^{*}\left(\psi_{x}\right)$ at some marked point $x$ is transformed into the class $h_{j}^{*}\left(\psi_{y}\right)$, where $y$ is the image of $x$ once we have contracted the unstable parts of the curve, while $j$ is the part on which $y$ lies.

It follows from the lemma that the integrand in the integral (8) is a pullback from $\overline{\mathbf{A}}_{1} \times \cdots \times \overline{\mathbf{A}}_{c}$. In particular, the integral vanishes if $\operatorname{dim} \mathrm{DIV}>\sum \operatorname{dim} \overline{\mathbf{A}}_{j}$.

(5) Splitting the integral.

Now assume that $\operatorname{dim} \mathrm{DIV}=\sum \operatorname{dim} \overline{\mathbf{A}}_{j}$

Then we can split the integral (8) into a product of similar integrals over $\overline{\mathbf{A}}_{1}, \ldots, \overline{\mathbf{A}}_{c}$ with the $\psi$-classes assigned as explained in step (4).

The total number of $\psi$-classes in these integrals equals $\sum d_{i}-1$, because we replaced one of the $\psi$-classes by the boundary divisors.

This completes the proof of Proposition 4.1.

\footnotetext{
${ }^{5}$ The dimension of DIV is equal to the number of marked points on $S$ (other than $0, \infty$ and the node) minus 2. The dimension of a DR-space is equal to the number of marked points on $S$ (other than 0 and $\infty$ ) minus 1 . The total number of marked points on $S$ does not change as we separate the parts, but each part that does not vanish contributes an additional "minus 1". Thus the dimension of $\overline{\mathbf{A}}_{1} \times \cdots \times \overline{\mathbf{A}}_{c}$ equals the number of marked points on $S$ minus $c$.
} 
Proposition 4.1 constitutes the recursive step of our algorithm. To make things precise we must add two comments.

(a) The condition $\left|k_{i}\right|>g \sum d_{j}$ is easily seen to be still satisfied for the smaller DR-spaces. Indeed, an index $k$ of a zero or a pole can decrease only by "annihilating" one or several black squares. For instance, in Figure 4 the initial index $k$ has become equal to $k-1$ on part IV by annihilating one square black dot. Thus $\sum d_{j}$ decreases by 1 , while $\left|k_{i}\right|$ decreases by at most $p \leq g$.

(b) The restriction of Witten's class (more precisely, of $h^{*}\left(c_{W}\right)$ ) on the components is obtained by using the factorization rules. This involves some choices or remainders modulo $r$. For example, in Figure 4, we will have to sum over $r-1$ possibilities of the remainders at the nodes connecting parts III and IV. The remainders assigned to the square dots are equal to 0 since they are forgotten under the map $h$.

Proof of Theorem 1 Assume we want to find the value of an integral over $\overline{\mathcal{M}}_{g, n}$ involving Witten's class and powers of the $\psi$-classes. First, using the above lemmas we can get rid of the $\psi$-classes and reduce the problem to computing the integral of Witten's class over DR-spaces. Comparing the degree of Witten's class

$$
\operatorname{deg} c_{W}=\frac{(r-2)(g-1)+\sum a_{i}}{r} \leq \frac{(r-2)(n+g-1)}{r}
$$

to the dimension of a DR-cycle

$$
\operatorname{dim}=2 g-3+n+p \geq 2 g-3+n,
$$

we see that such an integral can be nonzero only in 2 cases: either for genus 0 or for DR-cycles of codimension 1 in genus 1 . The genus zero integrals are well-known, while the case of double ramification divisors in genus 1 was treated in Section 3.

Remark 4.3 Our first goal was to compute the integral of $c_{W} \psi_{1}^{d_{1}} \ldots \psi_{n}^{d_{n}}$ over the moduli spaces $\overline{\mathcal{M}}_{g, n}$, but we actually computed such integrals over all DR-cycles satisfying the strange-looking condition $\left|k_{i}\right|>g \sum d_{j}$. This generalization is unavoidable if we want to make the algorithm work. It is easy to see that even if we start with an integral over a moduli space the algorithm can immediately lead us to integrals over DR-cycles.

\section{References}

[1] D Abramovich, T J Jarvis, Moduli of twisted spin curves, Proc. Amer. Math. Soc. 131 (2003) 685-699 MR1937405

Geometry 8 Topology, Volume 12 (2008) 
[2] L Chen, Y Li, K Liu, Localization, Hurwitz numbers and the Witten conjecture arXiv: math/0609263

[3] A Chiodo, Stable twisted curves and their $r$-spin structures, to appear in Annales de l'Institut Fourier arXiv:math/0603687

[4] A Chiodo, The Witten top Chern class via K-theory, J. Algebraic Geom. 15 (2006) 681-707 MR2237266

[5] C Faber, R Pandharipande, Relative maps and tautological classes, J. Eur. Math. Soc. (JEMS) 7 (2005) 13-49 MR2120989

[6] C Faber, S Shadrin, D Zvonkine, Tautological relations and the $r$-spin Witten conjecture arXiv:math/0612510

[7] E-N Ionel, Topological recursive relations in $H^{2 g}\left(\mathfrak{M}_{g, n}\right)$, Invent. Math. 148 (2002) 627-658 MR1908062

[8] T J Jarvis, Geometry of the moduli of higher spin curves, Internat. J. Math. 11 (2000) 637-663 MR1780734

[9] ME Kazarian, KP hierarchy and Hodge integrals (2007) available on http:/www.mi.ras.ru/ kazarian/

[10] M E Kazarian, S K Lando, An algebro-geometric proof of Witten's conjecture, J. Amer. Math. Soc. 20 (2007) 1079-1089 MR2328716

[11] Y-S Kim, K Liu, A simple proof of Witten conjecture through localization arXiv: math.AG/0508384

[12] M Kontsevich, Intersection theory on the moduli space of curves and the matrix Airy function, Comm. Math. Phys. 147 (1992) 1-23 MR1171758

[13] M Mirzakhani, Weil-Petersson volumes and intersection theory on the moduli space of curves, J. Amer. Math. Soc. 20 (2007) 1-23 MR2257394

[14] D Mumford, Towards an enumerative geometry of the moduli space of curves, from: "Arithmetic and geometry, Vol. II", Progr. Math. 36, Birkhäuser, Boston (1983) 271-328 MR717614

[15] A Okounkov, R Pandharipande, Gromov-Witten theory, Hurwitz numbers, and Matrix models, $I$ arXiv:math.AG/0101147

[16] A Polishchuk, Witten's top Chern class on the moduli space of higher spin curves, from: "Frobenius manifolds", Aspects Math. E36, Vieweg, Wiesbaden (2004) 253-264 MR2115773

[17] A Polishchuk, A Vaintrob, Algebraic construction of Witten's top Chern class, from: "Advances in algebraic geometry motivated by physics (Lowell, MA, 2000)", Contemp. Math. 276, Amer. Math. Soc. (2001) 229-249 MR1837120

[18] S V Shadrin, Geometry of meromorphic functions and intersections on moduli spaces of curves, Int. Math. Res. Not. (2003) 2051-2094 MR1994776 
[19] E Witten, Two-dimensional gravity and intersection theory on moduli space, from: "Surveys in differential geometry (Cambridge, MA, 1990)", Lehigh Univ., Bethlehem, PA (1991) 243-310 MR1144529

[20] E Witten, Algebraic geometry associated with matrix models of two-dimensional gravity, from: "Topological methods in modern mathematics (Stony Brook, NY, 1991)", Publish or Perish, Houston, TX (1993) 235-269 MR1215968

Korteweg-de Vries Institute for Mathematics

Plantage Muidergracht 24, 1018 TV Amsterdam, The Netherlands

and

Institute of System Research

Nakhimovskii Prospekt 36-1, 117218 Moscow, Russia

Poncelet Laboratory, CNRS

and

Independent University of Moscow

Bolshoi Vlassievsky per, 11, 119002 Moscow, Russia

sergey.shadrin@gmail.com, dimitri.zvonkine@gmail.com

Proposed: Eleny Ionel

Seconded: Jim Bryan, Yasha Eliashberg
Received: 5 January 2006

Revised: 22 January 2008 